\title{
CIRCUMSTELLAR ENVIRONMENT OF THE B[e] STAR MWC 349 (V 1478 CYG)
}

\author{
R.V. YUDIN \\ Central Astronomical Observatory of the Russian Academy of Sciences at Pulkovo, \\ 196140 Saint-Petersburg, Russia
}

Quasi-simultaneous optical and IR photometric (BVRIJHK-bands) and optical polarimetric (RI-bands) observations were made at the $1 \mathrm{~m}$ telescope of the Assy Observatory, Institute of Astrophysics of Kazakhstan between October 1985 and August 1992. We used the photopolarimeter of the Pulkovo Observatory described by Bergner et al. (1988). Most of our measurements were done with an aperture of $26^{\prime \prime}$. We obtained 26 polarimetric measurements of MWC 349, mainly in the R band. Our data show significant variations in the polarization which has not been reported previously. The amplitude of the polarization parameters is: $\Delta \mathrm{P}=8 \%$ and $\Delta \mathrm{P} . \mathrm{A} .=15^{\circ}$. During 12-13.09.90 five polarimetric observations have been obtained and $\Delta \mathrm{P}=3 \%$ on a time scale of 7 hours was detected. We found strong photometric variability in all photometric bands. There is however no correlation between brightness and polarization in any photometric band.

\section{Interstellar polarization and reddening}

The interstellar polarization of the Cyg OB2 association was investigated by Whittet et al. (1992). For 12 out of 19 stars in about $15^{\prime}$ around MWC 349 we obtained P.A. $=100^{\circ} \pm 20^{\circ}$ with an average of interstellar polarization degree of about 3-4\%. If we adopt 06.5-B0 ZAMS for MWC349, $(B-V)_{0}=$ $-0^{m} .32, \mathrm{R}=3.1$ in the Cygnus region and use the observed color index $2^{m} .41<(B-V)_{\text {obs }}<3^{m} .31$, the expected range of total extinction is $8^{m} .5<A_{V}<10^{m} .7$. The average interstellar reddening component would therefore be $A_{V}(i s)<8^{m} .5$ and the additional variable extinction $\left(A_{V}(c s)>\right.$ $2^{m} .0$.) arises in the circumstellar envelope.

After removing the interstellar component the position angle of intrinsic polarization in MWC 349 is about P.A.(int.) $=172^{\circ} \pm 3^{\circ}$ and $5 \%<\mathrm{P}$ (int.) $<$ $13 \%$. If scattering in dust grains in the circumstellar disk of MWC 349 is the dominant mechanism of the observed polarization, the dust disk must be oriented approximately in the East-West direction (P.A. $=82^{\circ} \pm 3^{\circ}$ ).

\section{The circumstellar environment of MWC 349}

We have evidence for two circumstellar disks in MWC 349: an inner dust disk (P.A. $=82^{\circ}$, this paper) and a large-scale outer disk (P.A. $=100^{\circ}$, 
Cohen et al. 1985), which make an angle of $15^{\circ}-20^{\circ}$. Using the formulae from Dolginov et al. (1979), from the alignment of the geometrical sizes of the dust disk and its declination one expects a polarization degree of $10 \%$ $15 \%$, which is in good agreement with what is observed. However, the main question concerns the explanation of the large and rapid variations in the polarization degree. One could explain these variations in terms of episodic eclipses of direct (unpolarized) stellar light by dust clouds moving through the line of sight (Grinin et al. 1988). The increase of polarization degree during the eclipse can then be described by: $p(\lambda)=p_{0}(\lambda) 10^{0.4 \Delta m_{\lambda}}$, where $p_{0}(\lambda)$ is the intrinsic polarization of the star without eclipse and $m_{\lambda}$ is the decrease of star's brightness at the given $\lambda$. In our case the observed $\Delta \mathrm{P}$ must correspond to a decrease in brightness of $>2^{m} .0$, which contradicts the observations. This means that the polarimetric changes must be due to variable physical conditions in the gas disk. The observed time scale of variability allows a rough estimate for the size of the circumstellar region where the polarization variability occurs. The average size derived from the several observed polarimetric variations is about $20-40 \mathrm{AU}$. Note that such sizes of a circumstellar shell are comparable to regions of masers. Maser features originate in two spots which arise from clumps of high-density ionized gas located in the near part of the gas disk surface (Planesas et al. 1992). Martin-Pintado et al. (1989) have calculated an upper limit for the size of the maser region of $<410^{14} \mathrm{~cm}^{-3}$ ( $\left.<27 \mathrm{AU}\right)$ which is close to what is observed. One can suppose that the variability of the polarization degree is associated with the presence of these clumps. Using the size and electron density of this region we estimate the polarization degree to be about $7 \%-8 \%$.

One can propose the following model for the explanation of the polarimetric behavior of MWC 349: during the eclipse of direct starlight by inhomogeneous dust condensations in the disk plane, the electron density in the dense clumps has changed. The speed by which the Strömgren sphere can respond to a change in the Lyman-continuum luminosity is set by the recombination time scale, $t_{\text {rec }}=10^{5}$ (yrs.) $/ \mathrm{Ne}$ (Thum et al. 1992).

For the densities of interest here $\left(\mathrm{Ne}>10^{8} \mathrm{~cm}^{-3}\right.$, Hamann and Simon, $1986,1988), t_{\text {rec }}$ is 8 hours or less, which is close to the observed time scale.

Bergner, Yu.K., et.al.: 1988, Izv.GAO AN SSSR, 205, 142 (in Russian)

Cohen, M., Biegning, J.H., Dreher, J.W., Welch, W.J.: 1985, Astrophys. J. 292, 249

Dolginov, A.Z., Gnedin, Yu.N., Silant'ev, N.A.: Propagation and polarization of radiation in Space, Moskow, Nauka, 1979 (in russian)

Grinin, V.P., Kiselev, N.N., Minikulov, N., Chernova, G.: 1988, Sov. Astr. Lett. 14, 514

Hamann, F., Simon, M.: 1986, Astrophys. J. 311, 909

Hamann, F., Simon, M.: 1988, Astrophys. J. 327, 876

Martin-Pintado, J., Thum, C., Bachiller, R.: 1989, Astron. Astrophys. , L9

Planesas, P., Martin-Pintado, J., Serabyn, E.: 1992, Astrophys. J. 386, L23

Thum, C., Martin-Pintado, J., Bachiller, R.: 1992, Astron. Astrophys. 256, 507

Whittet, D.C.B., et.al: 1992, Astrophys. J. 386, 562 